\title{
Highly efficient production of L-histidine from glucose by metabolically engineered Escherichia coli
}

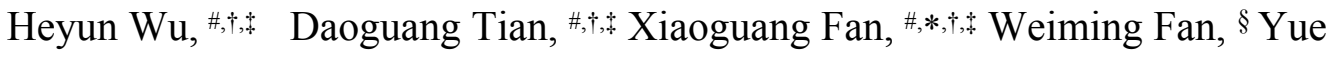

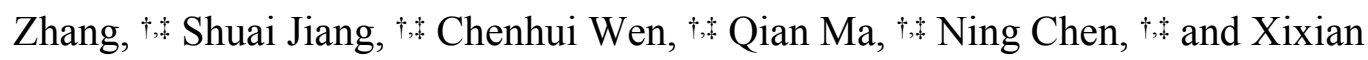
$\mathrm{Xie}^{*, \dagger,+}$
\end{abstract}

Table S1 Oligonucleotide primers used for gene cloning and chromosomal editing

\begin{tabular}{|c|c|}
\hline Primers & Sequence (5'-3') \\
\hline UP-lacI-S & GCAAAGAGGGTTACGGACAGA \\
\hline UP-lacI-A & GATTGCCCTTCACCGCCTTGTTTGCCCGCCAGTTGT \\
\hline DN-lacI-S & ACAACTGGCGGGCAAACAAGGCGGTGAAGGGCAATC \\
\hline DN-lacI-A & GCGCAACTGTTGGGAAGG \\
\hline UP-hisL-S & TCAATAATGATAACGACATGCTGC \\
\hline \multirow[t]{2}{*}{ UP-hisL-A } & AATTGTTATCCGCTCACAATTCCACACATTATACGAGCCGGATGAT \\
\hline & TAATTGTCAATGTTTAATGAATGAGCGGGGA \\
\hline \multirow[t]{2}{*}{ DN-hisL-S } & TCCGGCTCGTATAATGTGTGGAATTGTGAGCGGATAACAATTTCAC \\
\hline & ACAGGAAACAGACCATGACAGACAACACTCGTTTACGC \\
\hline DN-hisL-A & ACGGGTCAGCAGTTTGTCGA \\
\hline UP-hisL-hisG-S & GCGTTCTGCGGGTATCTTTG \\
\hline \multirow[t]{2}{*}{ UP-hisL-hisG-A } & AATTGTTATCCGCTCACAATTCCACACATTATACGAGCCGGATGAT \\
\hline & TAATTGTCAAAAAAGTCTCTGTGAATGTTTATTCAACT \\
\hline \multirow[t]{2}{*}{ DN-hisL-hisG-S } & TCCGGCTCGTATAATGTGTGGAATTGTGAGCGGATAACAATTTCAC \\
\hline & ACAGGAAACAGACCATGAGCTTTAACACAATCATTGACTG \\
\hline DN-hisL-hisG-A & TAAACACGTCCTGCACACCG \\
\hline $\mathrm{UP}-t d c D$-S & TATTCAAAACAGAAAAACCGTCAGT \\
\hline \multirow[t]{2}{*}{$\mathrm{UP}-t d c D-\mathrm{A}$} & AATTGTTATCCGCTCACAATTCCACACATTATACGAGCCGGATGAT \\
\hline & TAATTGTCAAGGCAAATCGCGAAGAAGTACAG \\
\hline \multirow[t]{2}{*}{$h i s G^{*} \mathrm{~S}$} & TCCGGCTCGTATAATGTGTGGAATTGTGAGCGGATAACAATTTCAC \\
\hline & ACAGGAAACAGACCATGTTGAAAATCGCTGTCCCA \\
\hline \multirow[t]{2}{*}{ his $G^{*}-\mathrm{A}$} & CACCGACAAACAACAGATAAAACGAAAGGCCCAGTCTTTCGACTG \\
\hline & AGCCTTTCGTTTTATTTGCTAGTTCTGCGCGTGCAAAA \\
\hline \multirow[t]{2}{*}{$\mathrm{DN}-t d c D-\mathrm{S}$} & AAAGACTGGGCCTTTCGTTTTATCTGTTGTTTGTCGGTGAACGCTCT \\
\hline & CCTGAGTAGGACAAATTGAATAATCGCTCTAACTCCTGTG \\
\hline $\mathrm{DN}-t d c D-\mathrm{A}$ & CGCCCTGGTTATGGGTTTT \\
\hline $\mathrm{UP}-y l b E-\mathrm{S}$ & ACCCAACCTTACGCAACCAG \\
\hline UP-ylbE-A & AATTGTTATCCGCTCACAATTCCACACATTATACGAGCCGGATGAT \\
\hline
\end{tabular}




\begin{tabular}{|c|c|}
\hline \multirow{3}{*}{$\mathrm{DN}-y l b E-\mathrm{S}$} & TAATTGTCAATTGTTCGATAACCGCAGCAT \\
\hline & AAAGACTGGGCCTTTCGTTTTATCTGTTGTTTGTCGGTGAACGCTCT \\
\hline & CCTGAGTAGGACAAATCGCTGGCGTGCTTTGAA \\
\hline $\mathrm{DN}-y l b E-\mathrm{A}$ & GGCGTAACTCAGCAGGCAG \\
\hline $\mathrm{UP}-i l v G-\mathrm{S}$ & ACCGAGGAGCAGACAATGAATAA \\
\hline \multirow[t]{2}{*}{$\mathrm{UP}-i l v G-\mathrm{A}$} & AATTGTTATCCGCTCACAATTCCACACATTATACGAGCCGGATGAT \\
\hline & TAATTGTCAAGGTGATGGCAACAACAGGGA \\
\hline \multirow[t]{2}{*}{$\mathrm{DN}-i l v G-\mathrm{S}$} & AAAGACTGGGCCTTTCGTTTTATCTGTTGTTTGTCGGTGAACGCTCT \\
\hline & CCTGAGTAGGACAAATCTATCTACGCGCCGTTGTTGT \\
\hline $\mathrm{DN}-i l v G-\mathrm{A}$ & GCGCTGGCTAACATGAGGAA \\
\hline $\mathrm{UP}-y g h X-\mathrm{S}$ & TTTGTTCTCTTCGACCTGATGAC \\
\hline \multirow[t]{2}{*}{$\mathrm{UP}-y g h X-\mathrm{A}$} & AATTGTTATCCGCTCACAATTCCACACATTATACGAGCCGGATGAT \\
\hline & TAATTGTCAACTGTTCTACGTTGCGCTTTTT \\
\hline \multirow[t]{2}{*}{ his $D-\mathrm{S}$} & TCCGGCTCGTATAATGTGTGGAATTGTGAGCGGATAACAATTTCAC \\
\hline & ACAGGAAACAGACCATGAGCTTTAACACAATCATTGACT \\
\hline \multirow[t]{2}{*}{ his D-A } & GCCCCAAGGGGTTATGCTAGCCTACAAATTGAGTTATGTTCATTTA \\
\hline & AATATGATGTTGTTCAG TCATGCTTGCTCCTTAAGGG \\
\hline \multirow[t]{2}{*}{$\mathrm{DN}-y g h X-\mathrm{S} 1$} & CTGAACAACATCATATTTAAATGAACATAACTCAATTTGTAGGCTA \\
\hline & GCATAACCCCTTGGGGC GTCATAGTAATCCAGCAACTCTTGTG \\
\hline DN-yghX-A & GAGCAGGTATTTACGTGAACCG \\
\hline $\mathrm{UP}-h i s C B-\mathrm{S}$ & ATTTCGTGGCTTCTGATTTGCT \\
\hline \multirow[t]{2}{*}{$\mathrm{UP}-h i s C B-A$} & AGTTGCTGGATTACTATGACCCTAGAAGAAATCAACCAGCGCATC \\
\hline & AGAAAGTCTCCTGTGCATTTACAGCACTCCTTTCGACGAG \\
\hline \multirow[t]{2}{*}{$\mathrm{DN}-y g h X-\mathrm{S} 2$} & ATGCACAGGAGACTTTCTGATGCGCTGGTTGATTTCTTCTAGGGTC \\
\hline & ATAGTAATCCAGCAACT GTCATAGTAATCCAGCAACTCTTGTG \\
\hline UP-hisHAFI-S & GTGACCGTTACGCTCACGTAGT \\
\hline \multirow[t]{2}{*}{ UP-hisHAFI-A } & CACCGACAAACAACAGATAAAACGAAAGGCCCAGTCTTTCGACTG \\
\hline & AGCCTTTCGTTTTATTTGTCACTGATGCCGTTTACGCA \\
\hline \multirow[t]{2}{*}{$\mathrm{DN}-y g h X-\mathrm{S} 3$} & AAAGACTGGGCCTTTCGTTTTATCTGTTGTTTGTCGGTGAACGCTCT \\
\hline & CCTGAGTAGGACAAAT GTCATAGTAATCCAGCAACTCTTGTG \\
\hline UP-trpR-S & ATGGCGATTGCTCGTCAG \\
\hline UP-trpR-A & CGCCACAAAGCCATTGAGGTGCGGATCAGTAACGACG \\
\hline $\operatorname{prs} A-\mathrm{S}$ & CGTCGTTACTGATCCGCACCTCAATGGCTTTGTGGCG \\
\hline $\operatorname{prs} A-\mathrm{A}$ & CGGGTATTTGTAGGACGGATAAATGGCAGGTGAAGGAGGC \\
\hline $\mathrm{DN}-\operatorname{trp} R-\mathrm{S}$ & GCCTCCTTCACCTGCCATTTATCCGTCCTACAAATACCCG \\
\hline $\mathrm{DN}-\operatorname{trp} R-\mathrm{A}$ & CGCCGTTTACTTCCAGAGG \\
\hline $\mathrm{UP}-p u r R-\mathrm{S}$ & TGGCGAAAGCATGACAGC \\
\hline UP-purR-A & GTGAGACGCTGAATAAGGAGTGCATTTCACTCCAGACCCTAACTTC \\
\hline DN-purR-S & GAAGTTAGGGTCTGGAGTGAAATGCACTCCTTATTCAGCGTCTCAC \\
\hline DN-purR-A & TCAGGAAGTGTTCAACGGTG \\
\hline $\mathrm{UP}-$ yeep-S & GGTCAGGAGGTAACTTATCAGCG \\
\hline \multirow[t]{2}{*}{ UP-yeep-A } & AATTGTTATCCGCTCACAATTCCACACATTATACGAGCCGGATGAT \\
\hline & TAATTGTCAAATGGCAGGGCTCCGTTTT \\
\hline$z w f-\mathrm{S}$ & TCCGGCTCGTATAATGTGTGGAATTGTGAGCGGATAACAATTTCAC \\
\hline
\end{tabular}




\begin{tabular}{|c|c|}
\hline \multirow{3}{*}{$z w f-\mathrm{A}$} & ACAGGAAACAGACCATGGCGGTAACGCAAACAG \\
\hline & CACCGACAAACAACAGATAAAACGAAAGGCCCAGTCTTTCGACTG \\
\hline & AGCCTTTCGTTTTATTTGTTACTCAAACTCATTCCAGGAACG \\
\hline \multirow[t]{2}{*}{ DN-yeep-S } & AAAGACTGGGCCTTTCGTTTTATCTGTTGTTTGTCGGTGAACGCTCT \\
\hline & CCTGAGTAGGACAAATGAACTGGATTTTCTTCTGAACCTGT \\
\hline DN-yeep-A & ACGATGTCAGCAGCCAGCA \\
\hline $\mathrm{UP}-y j i V-\mathrm{S}$ & GACTGTGGAAGCCCTGTATACG \\
\hline \multirow[t]{2}{*}{$\mathrm{UP}-y j i V-\mathrm{A}$} & AATTGTTATCCGCTCACAATTCCACACATTATACGAGCCGGATGAT \\
\hline & TAATTGTCAACTGTCCCTTGTCGACTGTCTGT \\
\hline \multirow[t]{2}{*}{ gnd-S } & TCCGGCTCGTATAATGTGTGGAATTGTGAGCGGATAACAATTTCAC \\
\hline & ACAGGAAACAGACCATGTCCAAGCAACAGATCGGC \\
\hline \multirow[t]{2}{*}{ gnd-A } & CACCGACAAACAACAGATAAAACGAAAGGCCCAGTCTTTCGACTG \\
\hline & AGCCTTTCGTTTTATTTGTTAATCCAGCCATTCGGTATGG \\
\hline \multirow[t]{2}{*}{$\mathrm{DN}-y j i V-\mathrm{S}$} & AAAGACTGGGCCTTTCGTTTTATCTGTTGTTTGTCGGTGAACGCTCT \\
\hline & CCTGAGTAGGACAAAT TGAACGAGTTTATTCTGCCGG \\
\hline DN-yjiV-A & TGGCGACATTCCCTTCCTT \\
\hline UP-pgi-S & ACGCTAACGGCACTAAAACCA \\
\hline UP-pgi-A & GCTGACCGTTAGTGCCTGGTGCGTTGACTTCCGGCATTA \\
\hline DN-pgi-S & TAATGCCGGAAGTCAACGCACCAGGCACTAACGGTCAGC \\
\hline DN-pgi-A & TCTTTATCATCTTTCAGCTCTGGC \\
\hline $\mathrm{UP}-m a z G-\mathrm{S}$ & GGAGAGCCGAAAGATAAGGAAGT \\
\hline UP-mazG-A & CAACAATACGCTCCACTTCGCTCGTAGGTTTCTTCAAGGGTGTAA \\
\hline $\mathrm{DN}-m a z G-\mathrm{S}$ & TTACACCCTTGAAGAAACCTACGAGCGAAGTGGAGCGTATTGTTG \\
\hline $\mathrm{DN}-m a z G-\mathrm{A}$ & GAATGAAACGCTCGTAGTGCC \\
\hline UP-ushA-S & TTCTGACTGAAGCAGCGGATAA \\
\hline UP-ushA-A & ACTCACATCCAGCGGCGATCATACGCCAGAGCAGTTTCAC \\
\hline $\mathrm{DN}-u s h A-\mathrm{S}$ & GTGAAACTGCTCTGGCGTATGATCGCCGCTGGATGTGAGT \\
\hline $\mathrm{DN}-u s h A-\mathrm{A}$ & TGGTGGCAGTGAACGGTGA \\
\hline UP-surE-S & TGTCGCAACCACCGAAGAA \\
\hline UP-surE-A & GCTTACCATTGCGTGCCAACAGCAAACTCACGCAAGGC \\
\hline DN-surE-S & GCCTTGCGTGAGTTTGCTGTTGGCACGCAATGGTAAGC \\
\hline DN-surE-A & TAATCCGTTCAACCGAGCAAA \\
\hline $\mathrm{UP}-y j j G-\mathrm{S}$ & AACCAGGGCGAGCGTTATC \\
\hline $\mathrm{UP}-y j j G-\mathrm{A}$ & GAAACGGTCCAGGTGGGCCAGTCCCACTTCATTATTCCACC \\
\hline $\mathrm{DN}-y j j G-\mathrm{S}$ & GGTGGAATAATGAAGTGGGACTGGCCCACCTGGACCGTTTC \\
\hline $\mathrm{DN}-y j j G-\mathrm{A}$ & TCAGGCAGCAGTCCACCG \\
\hline UP-yrfG-S & GCGACGAAGGCAGCGAT \\
\hline $\mathrm{UP}-y r f G-\mathrm{A}$ & GGATGGCGTTGATACTGTTTCTCGCCGTCCATATCCAGCAGAA \\
\hline $\mathrm{DN}-y r f G-\mathrm{S}$ & TTCTGCTGGATATGGACGGCGAGAAACAGTATCAACGCCATCC \\
\hline $\mathrm{DN}-y r f G-\mathrm{A}$ & GCGATGTAATTGGTCATGTTGC \\
\hline UP-amn-S & TGGAGTCGGTTATCAGGTTGC \\
\hline UP-amn-A & AACGGCGGCTCATTAAAGGTGAGTAGGGCGAGTAATCGTGGT \\
\hline $\mathrm{DN}-a m n-\mathrm{S}$ & ACCACGATTACTCGCCCTACTCACCTTTAATGAGCCGCCGTT \\
\hline DN-amn-A & CAGCAGCATAGATTTCTACACCGA \\
\hline
\end{tabular}




\begin{tabular}{|c|c|}
\hline $\mathrm{UP}-y g d H-\mathrm{S}$ & TAGCCAATCATTCTTACTCCCTTT \\
\hline $\mathrm{UP}-y g d H-\mathrm{A}$ & TCCGGTCCTAATGCCTCCAGCATATCGGTAACGACAATAAACAG \\
\hline $\mathrm{DN}-y g d H-\mathrm{S}$ & CTGTTTATTGTCGTTACCGATATGCTGGAGGCATTAGGACCGGA \\
\hline DN-ygdH-A & GAAGCCGTTCCACAGCATTG \\
\hline UP-rph-S & ATAGCGCAGGGTACATTCCACT \\
\hline \multirow[t]{2}{*}{ UP-rph-A } & AATTGTTATCCGCTCACAATTCCACACATTATACGAGCCGGATGAT \\
\hline & TAATTGTCAATTCTTCAATAGAGGCGGTACA \\
\hline \multirow[t]{2}{*}{ pur $H-\mathrm{S}$} & TCCGGCTCGTATAATGTGTGGAATTGTGAGCGGATAACAATTTCAC \\
\hline & ACAGGAAACAGACCATGCAACAACGTCGTCCAGTC \\
\hline \multirow[t]{2}{*}{ purH-A } & CACCGACAAACAACAGATAAAACGAAAGGCCCAGTCTTTCGACTG \\
\hline & AGCCTTTCGTTTTATTTGTTAATGGCGGAAGTGGCG \\
\hline \multirow[t]{2}{*}{$\mathrm{DN}-r p h-\mathrm{S}$} & AAAGACTGGGCCTTTCGTTTTATCTGTTGTTTGTCGGTGAACGCTCT \\
\hline & CCTGAGTAGGACAAATTGCCGCAGAGACCGACAT \\
\hline DN-rph-A & ACAGCGGTTGTGGTGGCA \\
\hline $\mathrm{UP}-y c j V-\mathrm{S}$ & GAATGCGCCGACGATTTTAG \\
\hline \multirow[t]{2}{*}{$\mathrm{UP}-y c j V$-A } & AATTGTTATCCGCTCACAATTCCACACATTATACGAGCCGGATGAT \\
\hline & TAATTGTCAAGCCGACAAACACGATGAACTC \\
\hline \multirow[t]{2}{*}{ purA-S } & TCCGGCTCGTATAATGTGTGGAATTGTGAGCGGATAACAATTTCAC \\
\hline & ACAGGAAACAGACCATGGGTAACAACGTCGTCGTACT \\
\hline \multirow[t]{2}{*}{ purA-A } & CACCGACAAACAACAGATAAAACGAAAGGCCCAGTCTTTCGACTG \\
\hline & AGCCTTTCGTTTTATTTGTTACGCGTCGAACGGGTC \\
\hline \multirow[t]{2}{*}{$\mathrm{DN}-y c j V-\mathrm{S}$} & AAAGACTGGGCCTTTCGTTTTATCTGTTGTTTGTCGGTGAACGCTCT \\
\hline & CCTGAGTAGGACAAATCCACCATGATCTACGTGACCC \\
\hline $\mathrm{DN}-y c j V-\mathrm{A}$ & CACCAGCACGGACCACTAACT \\
\hline UP-yjiP-S & GCCATACCGCCAGCAAGAT \\
\hline \multirow[t]{2}{*}{$\mathrm{UP}-y j i P-\mathrm{A}$} & AATTGTTATCCGCTCACAATTCCACACATTATACGAGCCGGATGAT \\
\hline & TAATTGTCAAGCAGATATTCCCCTTTCCACC \\
\hline \multirow[t]{2}{*}{ purB-S } & TCCGGCTCGTATAATGTGTGGAATTGTGAGCGGATAACAATTTCAC \\
\hline & ACAGGAAACAGACCATGGAATTATCCTCACTGACCGC \\
\hline \multirow[t]{2}{*}{ purB-A } & CACCGACAAACAACAGATAAAACGAAAGGCCCAGTCTTTCGACTG \\
\hline & AGCCTTTCGTTTTATTTGTTATTTCAGCTCATCAACCATCGT \\
\hline \multirow[t]{2}{*}{ DN-yjiP-S } & AAAGACTGGGCCTTTCGTTTTATCTGTTGTTTGTCGGTGAACGCTCT \\
\hline & CCTGAGTAGGACAAATGACGGATGACAAACGCAAAGC \\
\hline $\mathrm{DN}-y j i P-\mathrm{A}$ & AAAGGCGGATTTTTACTGTGGA \\
\hline UP-purF-S & GGCTGGTGAGCGATGTATTTG \\
\hline UP-purF-A & CACTGCTTTGGCGTCATCATTAGAAATCAAAGCCCAGCGTATC \\
\hline DN-purF-S & GATACGCTGGGCTTTGATTTCTAATGATGACGCCAAAGCAGTG \\
\hline DN-purF-A & AAAGGGTTTTGATTGAACAGGGTA \\
\hline UP-yjhE-S & GTCAGGCACTGGCGAAAGAT \\
\hline \multirow[t]{2}{*}{ UP-yjhE-A } & AATTGTTATCCGCTCACAATTCCACACATTATACGAGCCGGATGAT \\
\hline & TAATTGTCAACGCAAGCCATAAACCCACA \\
\hline \multirow[t]{2}{*}{$\operatorname{roc} G-\mathrm{S}$} & TCCGGCTCGTATAATGTGTGGAATTGTGAGCGGATAACAATTTCAC \\
\hline & ACAGGAAACAGACCATGTCAGCAAAGCAAGTCTCGA \\
\hline $\operatorname{roc} G-\mathrm{A}$ & CACCGACAAACAACAGATAAAACGAAAGGCCCAGTCTTTCGACTG \\
\hline
\end{tabular}




\begin{tabular}{|c|c|}
\hline \multirow{3}{*}{$\mathrm{DN}-y j h E-\mathrm{S}$} & AGCCTTTCGTTTTATTTGTTAGACCCATCCGCGGAAA \\
\hline & CTGGGCCTTTCGTTTTATCTGTTGTTTGTCGGTGAACGCTCTCCTGA \\
\hline & GTAGGACAAATTTCCGACATCGAAATGCGT \\
\hline $\mathrm{DN}-y j h E-\mathrm{A}$ & AGGCGTTGTTGTGGCAGATT \\
\hline UP-hisJ-S & AAAGATGATGCTGAACTGACGG \\
\hline UP-hisJ-A & AGTTCGTTATCTTCTTTACGCAGGGATTCAAATGGCGCATAGGTC \\
\hline DN-hisJ-S & GACCTATGCGCCATTTGAATCCCTGCGTAAAGAAGATAACGAACT \\
\hline DN-hisJ-A & GCCTCCGTCACCGTGTTTAG \\
\hline UP-aroP-S & CTGCCGCATACCATTATTCTTG \\
\hline UP-aroP-A & ATTCCTGGGGTCATCAGCATAATGTATTTACCCACGGCAGTCAG \\
\hline $\mathrm{DN}$-aroP-S & CTGACTGCCGTGGGTAAATACATTATGCTGATGACCCCAGGAAT \\
\hline DN-aroP-A & GCTGGGTGATTTCCATACTTCG \\
\hline UP-yjiT-S & AATAGTTGTTGCCGCCTGAGT \\
\hline \multirow[t]{2}{*}{ UP-yjiT-A } & AATTGTTATCCGCTCACAATTCCACACATTATACGAGCCGGATGAT \\
\hline & TAATTGTCAAAAAACAGGCAGCAAAGTCCC \\
\hline \multirow[t]{2}{*}{ lysE-S } & TCCGGCTCGTATAATGTGTGGAATTGTGAGCGGATAACAATTTCAC \\
\hline & ACAGGAAACAGACCATGGTGATCATGGAAATCTTCATTA \\
\hline \multirow[t]{2}{*}{ lysE-A } & CACCGACAAACAACAGATAAAACGAAAGGCCCAGTCTTTCGACTG \\
\hline & AGCCTTTCGTTTTATTTGCTAACCCATCAACATCAGTTTGATG \\
\hline \multirow[t]{2}{*}{$\arg O-S$} & TCCGGCTCGTATAATGTGTGGAATTGTGAGCGGATAACAATTTCAC \\
\hline & ACAGGAAACAGACCGTGTTTTCTTATTACTTTCAAGGTCTT \\
\hline \multirow[t]{2}{*}{$\arg O-A$} & CACCGACAAACAACAGATAAAACGAAAGGCCCAGTCTTTCGACTG \\
\hline & AGCCTTTCGTTTTATTTGCTAACTGAACAAGGCTTGTGCA \\
\hline \multirow[t]{2}{*}{$\mathrm{DN}-y j i T-\mathrm{S}$} & AAAGACTGGGCCTTTCGTTTTATCTGTTGTTTGTCGGTGAACGCTCT \\
\hline & CCTGAGTAGGACAAATAAGCACTACCTGTGAAGGGATGT \\
\hline DN-yjiT-A & CAGGGCTTCCACAGTCACAAT \\
\hline \multirow[t]{2}{*}{ gRNA-lacI-S } & AGTCCTAGGTATAATACTAGTGAATTACATTCCCAACCGCGGTTTT \\
\hline & AGAGCTAGAA \\
\hline \multirow[t]{2}{*}{ gRNA-lacI-A } & TTCTAGCTCTAAAACCGCGGTTGGGAATGTAATTCACTAGTATTAT \\
\hline & ACCTAGGACT \\
\hline \multirow[t]{2}{*}{ gRNA-hisL-A } & AGTCCTAGGTATAATACTAGTATCATCCTGACTAGTCTTTCGTTTTA \\
\hline & GAGCTAGAA \\
\hline \multirow[t]{2}{*}{ gRNA-hisL-A } & TTCTAGCTCTAAAACGAAAGACTAGTCAGGATGATACTAGTATTAT \\
\hline & ACCTAGGACT \\
\hline \multirow[t]{2}{*}{ gRNA-hisL-hisG-A } & AGTCCTAGGTATAATACTAGTCACCCAGCGCCTGATCGCGAGTTTT \\
\hline & AGAGCTAGAA \\
\hline \multirow[t]{2}{*}{ gRNA-hisL-hisG-A } & TTCTAGCTCTAAAACTCGCGATCAGGCGCTGGGTGACTAGTATTAT \\
\hline & ACCTAGGACT \\
\hline \multirow[t]{2}{*}{ gRNA- $t d c D-\mathrm{S}$} & AGTCCTAGGTATAATACTAGTAAAAGAGATGAATGAATTTCGTTTT \\
\hline & AGAGCTAGAA \\
\hline \multirow[t]{2}{*}{ gRNA-tdcD-A } & TTCTAGCTCTAAAACGAAATTCATTCATCTCTTTTACTAGTATTATA \\
\hline & CCTAGGACT \\
\hline \multirow[t]{2}{*}{ gRNA-ylbE-S } & AGTCCTAGGTATAATACTAGTACACTGGCTGGATGTGCAACGTTTT \\
\hline & AGAGCTAGAA \\
\hline
\end{tabular}




\begin{tabular}{|c|c|}
\hline gRNA-ylbE-A & $\begin{array}{l}\text { TTCTAGCTCTAAAACGTTGCACATCCAGCCAGTGTACTAGTATTAT } \\
\text { ACCTAGGACT }\end{array}$ \\
\hline gRNA-ilvG-S & $\begin{array}{l}\text { AGTCCTAGGTATAATACTAGTTATCGGCACTGACGCATTTCGTTTT } \\
\text { AGAGCTAGAA }\end{array}$ \\
\hline gRNA-ilvG-A & $\begin{array}{l}\text { TTCTAGCTCTAAAACGAAATGCGTCAGTGCCGATAACTAGTATTAT } \\
\text { ACCTAGGACT }\end{array}$ \\
\hline gRNA-yghX-S & $\begin{array}{l}\text { AGTCCTAGGTATAATACTAGTGGTGCCTGACGACCATAAAAGTTTT } \\
\text { AGAGCTAGAA }\end{array}$ \\
\hline gRNA-ygh $X$-A & $\begin{array}{l}\text { TTCTAGCTCTAAAACTTTTATGGTCGTCAGGCACCACTAGTATTAT } \\
\text { ACCTAGGACT }\end{array}$ \\
\hline gRNA-his1-S & $\begin{array}{l}\text { AGTCCTAGGTATAATACTAGTATGAACATAACTCAATTTGTGTTTT } \\
\text { AGAGCTAGAA }\end{array}$ \\
\hline gRNA-his1-A & $\begin{array}{l}\text { TTCTAGCTCTAAAACACAAATTGAGTTATGTTCATACTAGTATTAT } \\
\text { ACCTAGGACT }\end{array}$ \\
\hline gRNA-his2-S & $\begin{array}{l}\text { AGTCCTAGGTATAATACTAGTTGCGCTGGTTGATTTCTTCTGTTTTA } \\
\text { GAGCTAGAA }\end{array}$ \\
\hline gRNA-his2-A & $\begin{array}{l}\text { TTCTAGCTCTAAAACAGAAGAAATCAACCAGCGCAACTAGTATTA } \\
\text { TACCTAGGACT }\end{array}$ \\
\hline gRNA-trpR-S & $\begin{array}{l}\text { AGTCCTAGGTATAATACTAGTGATGGCAGAACAGCGTCACCGTTTT } \\
\text { AGAGCTAGAA }\end{array}$ \\
\hline gRNA-trpR-A & $\begin{array}{l}\text { AGTCCTAGGTATAATACTAGTGATGGCAGAACAGCGTCACCGTTTT } \\
\text { AGAGCTAGAA }\end{array}$ \\
\hline gRNA-purR-S & $\begin{array}{l}\text { AGTCCTAGGTATAATACTAGTAGAAACGCGCAACGCCGTGTGTTTT } \\
\text { AGAGCTAGAA }\end{array}$ \\
\hline gRNA-purR-A & $\begin{array}{l}\text { TTCTAGCTCTAAAACACACGGCGTTGCGCGTTTCTACTAGTATTAT } \\
\text { ACCTAGGACT }\end{array}$ \\
\hline gRNA-yеep-S & $\begin{array}{l}\text { AGTCCTAGGTATAATACTAGTACAGAATATTCGCGAAAAAAGTTTT } \\
\text { AGAGCTAGAA }\end{array}$ \\
\hline gRNA-yeep-A & $\begin{array}{l}\text { TTCTAGCTCTAAAACTTTTTTCGCGAATATTCTGTACTAGTATTATA } \\
\text { CCTAGGACT }\end{array}$ \\
\hline gRNA-yjiV-S & $\begin{array}{l}\text { AGTCCTAGGTATAATACTAGTGCGTAGTCGAAATTCTCAGCGTTTT } \\
\text { AGAGCTAGAA }\end{array}$ \\
\hline gRNA-yjiV-A & $\begin{array}{l}\text { TTCTAGCTCTAAAACGCTGAGAATTTCGACTACGCACTAGTATTAT } \\
\text { ACCTAGGACT }\end{array}$ \\
\hline gRNA-pgi-S & $\begin{array}{l}\text { AGTCCTAGGTATAATACTAGTTTCTGACCTCGGCCCATACAGTTTT } \\
\text { AGAGCTAGAA }\end{array}$ \\
\hline gRNA-pgi-A & $\begin{array}{l}\text { TTCTAGCTCTAAAACTGTATGGGCCGAGGTCAGAAACTAGTATTAT } \\
\text { ACCTAGGACT }\end{array}$ \\
\hline gRNA- $m a z G-\mathrm{S}$ & $\begin{array}{l}\text { AGTCCTAGGTATAATACTAGTACTGGGCGATCTGCTATTCCGTTTT } \\
\text { AGAGCTAGAA }\end{array}$ \\
\hline gRNA-mazG-A & $\begin{array}{l}\text { TTCTAGCTCTAAAACGGAATAGCAGATCGCCCAGTACTAGTATTAT } \\
\text { ACCTAGGACT }\end{array}$ \\
\hline gRNA-ushA-S & $\begin{array}{l}\text { AGTCCTAGGTATAATACTAGTCTGCATACCAATGATCATCAGTTTT } \\
\text { AGAGCTAGAA }\end{array}$ \\
\hline
\end{tabular}




\begin{tabular}{|c|c|}
\hline gRNA-ushA-A & $\begin{array}{l}\text { TTCTAGCTCTAAAACTGATGATCATTGGTATGCAGACTAGTATTAT } \\
\text { ACCTAGGACT }\end{array}$ \\
\hline gRNA-surE-S & $\begin{array}{l}\text { AGTCCTAGGTATAATACTAGTGCCCCCGATCGTAACCGCAGGTTTT } \\
\text { AGAGCTAGAA }\end{array}$ \\
\hline gRNA-surE-A & $\begin{array}{l}\text { TTCTAGCTCTAAAACCTGCGGTTACGATCGGGGGCACTAGTATTAT } \\
\text { ACCTAGGACT }\end{array}$ \\
\hline gRNA- $y r f G$-S & $\begin{array}{l}\text { AGTCCTAGGTATAATACTAGTTAACTATTTCTGGCAAAAGCGTTTT } \\
\text { AGAGCTAGAA }\end{array}$ \\
\hline gRNA- $y r f G$-A & $\begin{array}{l}\text { TTCTAGCTCTAAAACGCTTTTGCCAGAAATAGTTAACTAGTATTAT } \\
\text { ACCTAGGACT }\end{array}$ \\
\hline gRNA-yjjG-S & $\begin{array}{l}\text { AGTCCTAGGTATAATACTAGTTTTACCTTTGACTCATTCACGTTTTA } \\
\text { GAGCTAGAA }\end{array}$ \\
\hline gRNA- $y j j G$-A & $\begin{array}{l}\text { TTCTAGCTCTAAAACGTGAATGAGTCAAAGGTAAAACTAGTATTAT } \\
\text { ACCTAGGACT }\end{array}$ \\
\hline gRNA-amn-S & $\begin{array}{l}\text { AGTCCTAGGTATAATACTAGTTTGCCCTTTCTTGTGCTGGCGTTTTA } \\
\text { GAGCTAGAA }\end{array}$ \\
\hline gRNA-amn-A & $\begin{array}{l}\text { TTCTAGCTCTAAAACGCCAGCACAAGAAAGGGCAAACTAGTATTA } \\
\text { TACCTAGGACT }\end{array}$ \\
\hline gRNA-ygdH-S & $\begin{array}{l}\text { AGTCCTAGGTATAATACTAGTCGCTATCAATCGCGGTTTTCGTTTT } \\
\text { AGAGCTAGAA }\end{array}$ \\
\hline gRNA-ygdH-A & $\begin{array}{l}\text { TTCTAGCTCTAAAACGAAAACCGCGATTGATAGCGACTAGTATTAT } \\
\text { ACCTAGGACT }\end{array}$ \\
\hline gRNA-rph-S & $\begin{array}{l}\text { AGTCCTAGGTATAATACTAGTGGCTGGATCACCGCAGAGTAGTTTT } \\
\text { AGAGCTAGAA }\end{array}$ \\
\hline gRNA-rph-A & $\begin{array}{l}\text { TTCTAGCTCTAAAACTACTCTGCGGTGATCCAGCCACTAGTATTAT } \\
\text { ACCTAGGACT }\end{array}$ \\
\hline gRNA- $y c j V-\mathrm{S}$ & $\begin{array}{l}\text { AGTCCTAGGTATAATACTAGTCAAAGCACGCAATATAGCGAGTTTT } \\
\text { AGAGCTAGAA }\end{array}$ \\
\hline gRNA-ycjV-A & $\begin{array}{l}\text { TTCTAGCTCTAAAACTCGCTATATTGCGTGCTTTGACTAGTATTATA } \\
\text { CCTAGGACT }\end{array}$ \\
\hline gRNA-yjiP-S & $\begin{array}{l}\text { AGTCCTAGGTATAATACTAGTGTAAAGACCCGCGAAGGCGAGTTT } \\
\text { TAGAGCTAGAA }\end{array}$ \\
\hline gRNA-yjiP-A & $\begin{array}{l}\text { TTCTAGCTCTAAAACTCGCCTTCGCGGGTCTTTACACTAGTATTATA } \\
\text { CCTAGGACT }\end{array}$ \\
\hline gRNA-purF-S & $\begin{array}{l}\text { AGTCCTAGGTATAATACTAGTTGTGAATATGGGCACGAAACGTTTT } \\
\text { AGAGCTAGAA }\end{array}$ \\
\hline gRNA-purF-A & $\begin{array}{l}\text { TTCTAGCTCTAAAACGTTTCGTGCCCATATTCACAACTAGTATTAT } \\
\text { ACCTAGGACT }\end{array}$ \\
\hline gRNA-yjhE-S & $\begin{array}{l}\text { AGTCCTAGGTATAATACTAGTAATGCCGGGGATGGAGCGCCGTTTT } \\
\text { AGAGCTAGAA }\end{array}$ \\
\hline gRNA-yjhE-A & $\begin{array}{l}\text { TTCTAGCTCTAAAACGGCGCTCCATCCCCGGCATTACTAGTATTAT } \\
\text { ACCTAGGACT }\end{array}$ \\
\hline gRNA-hisJ-S & $\begin{array}{l}\text { AGTCCTAGGTATAATACTAGTATTTATTCTGACCTGACTGCGTTTTA } \\
\text { GAGCTAGAA }\end{array}$ \\
\hline
\end{tabular}




\begin{tabular}{ll}
\hline gRNA-his $J$-A & TTCTAGCTCTAAAACGCAGTCAGGTCAGAATAAATACTAGTATTAT \\
& ACCTAGGACT \\
gRNA-aroP-S & AGTCCTAGGTATAATACTAGTATTATCATGTTCTCGTTCGGGTTTTA \\
& GAGCTAGAA \\
gRNA-arop-A & TTCTAGCTCTAAAACCCGAACGAGAACATGATAATACTAGTATTAT \\
& ACCTAGGACT \\
gRNA-yjiT-S & AGTCCTAGGTATAATACTAGTAGGGATTATGAACGGCAATGGTTTT \\
& AGAGCTAGAA \\
gRNA-yjiT-A & TTCTAGCTCTAAAACCATTGCCGTTCATAATCCCTACTAGTATTATA \\
& CCTAGGACT \\
\hline
\end{tabular}

Table S2 Composition of trace element solution

\begin{tabular}{cc}
\hline Component & Concentration $(\mathbf{g} / \mathbf{L})$ \\
\hline $\mathrm{Na}_{2} \mathrm{MoO}_{4}$ & 2.5 \\
$\mathrm{NiCl}_{2}$ & 1.6 \\
$\mathrm{CaCl}_{2}$ & 10 \\
$\mathrm{CuSO}_{4}$ & 0.4 \\
$\mathrm{Al}_{2}\left(\mathrm{SO}_{4}\right)_{2}$ & 2.25 \\
$\mathrm{CoCl}_{2}$ & 1.8 \\
$\mathrm{ZnSO}_{4}$ & 0.5 \\
$\mathrm{H}_{3} \mathrm{BO}_{3}$ & 0.14 \\
\hline
\end{tabular}
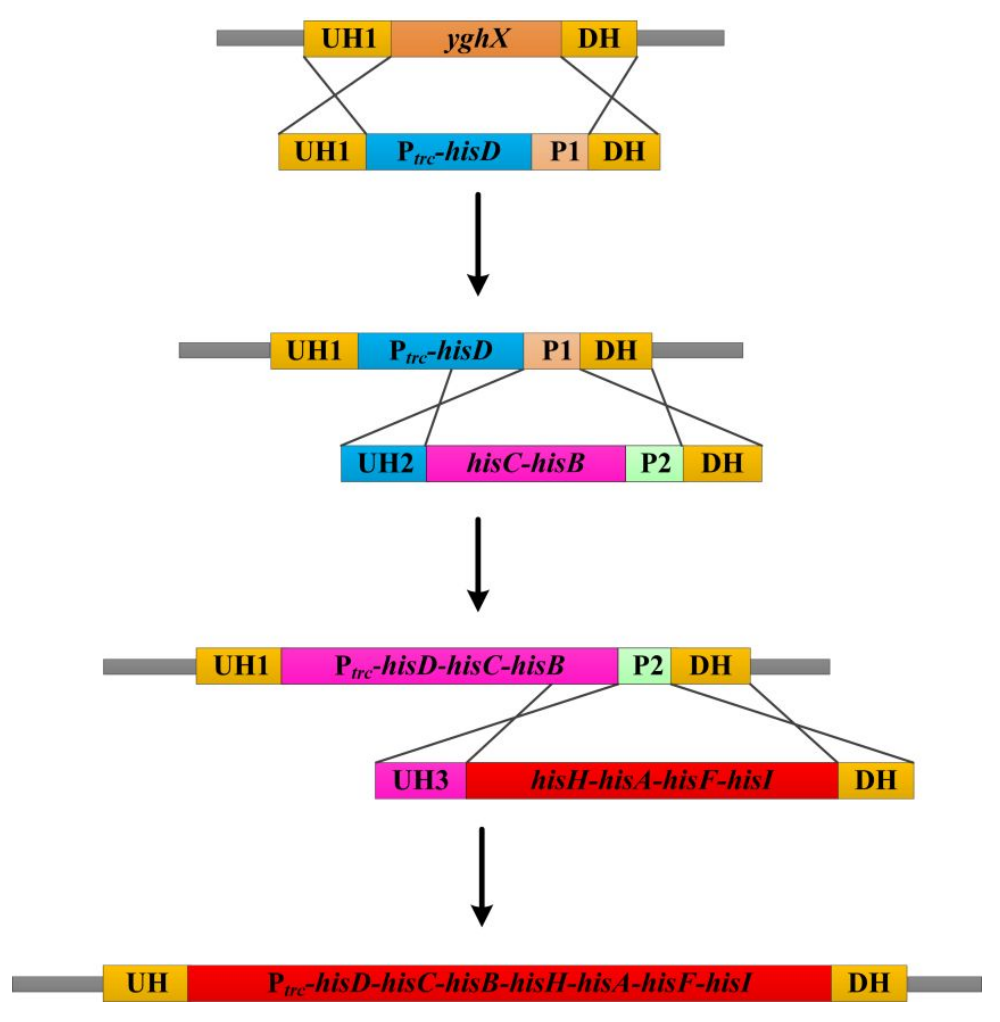
Figure S1 Strategy for the integration of the downstream his operon genes (hisD-hisC-hisB-hisH-hisA-hisF-hisI)

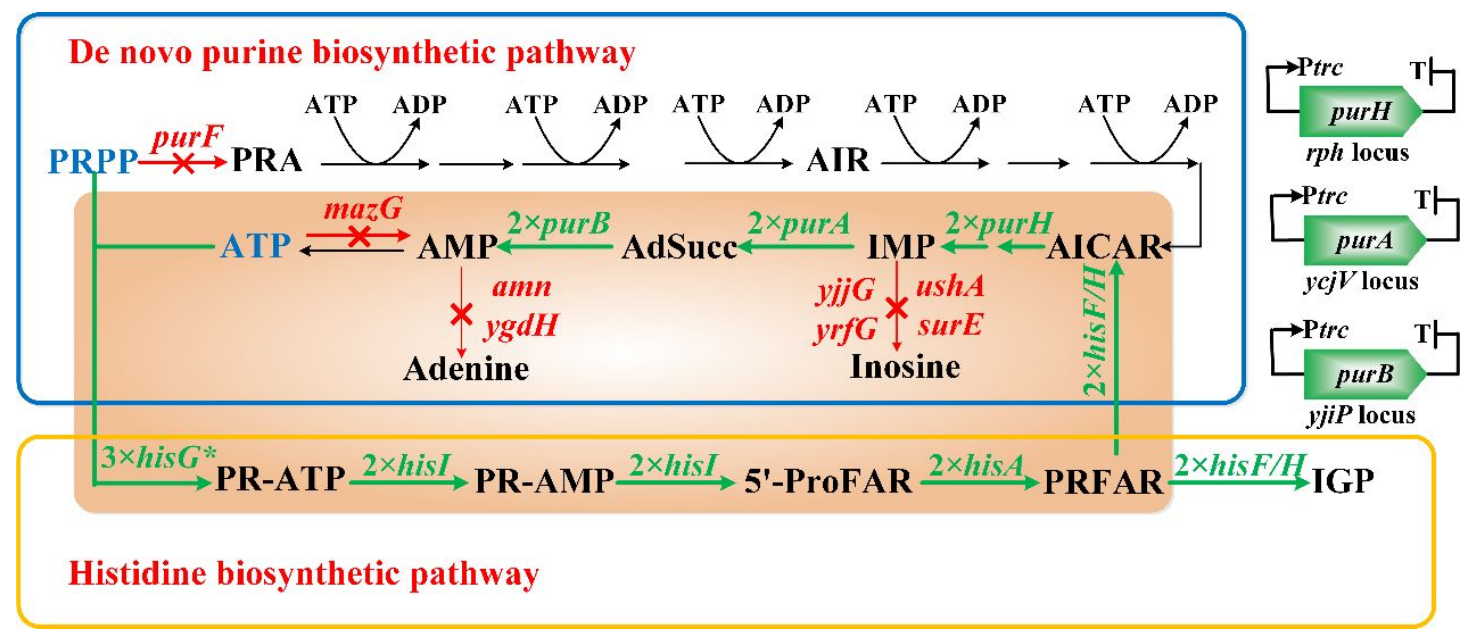

Figure S2 Strategies for rerouting the purine nucleotide biosynthetic pathway

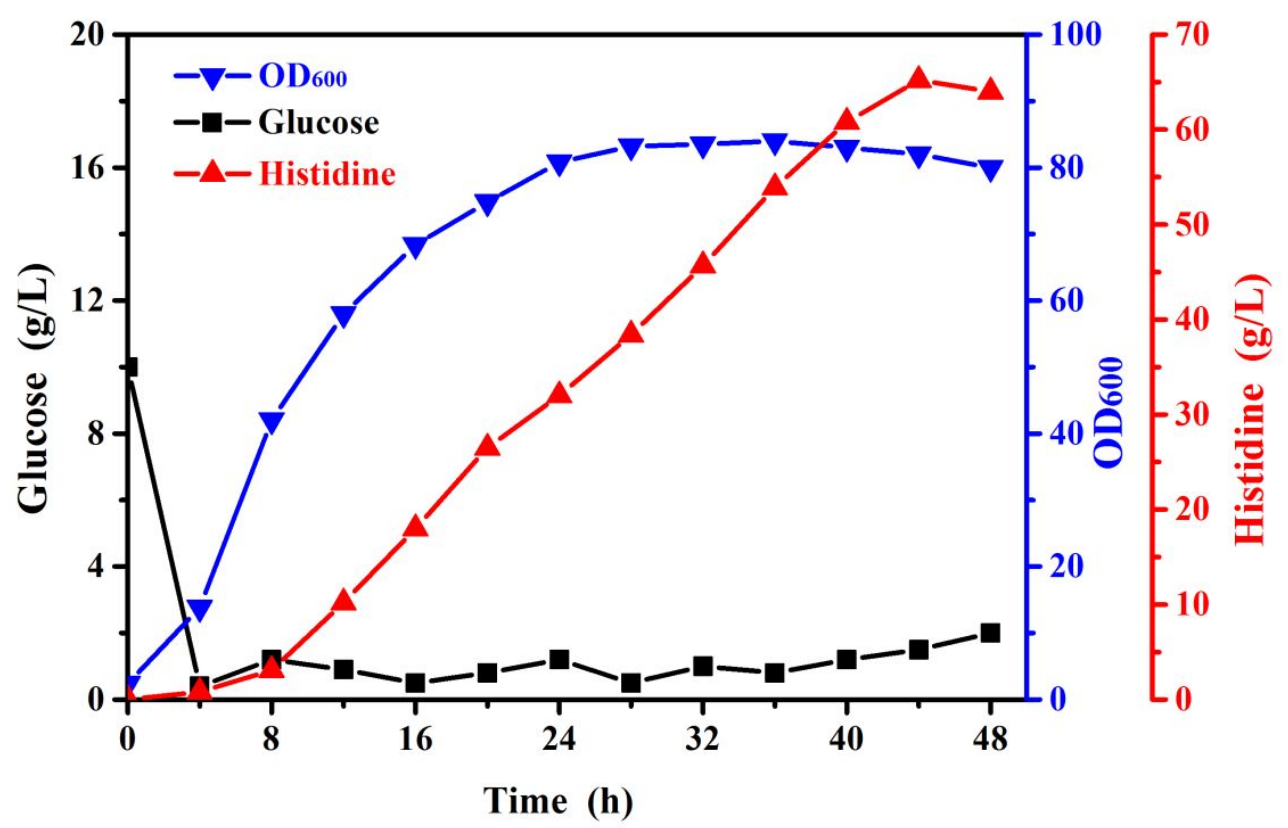

Figure S3 Fed-batch production of histidine in a 5 L bioreactor using strain HW6-3 (duplicate experiment) 